\title{
Electronic excitation spectrum of metallic carbon nanotubes
}

\author{
S. Sapmaz, P. Jarillo-Herrero, J. Kong, C. Dekker, L. P. Kouwenhoven, and H. S. J. van der Zant \\ Kavli Institute of Nanoscience, Delft University of Technology, P.O. Box 5046, 2600 GA Delft, The Netherlands
}

(Received 17 December 2004; published 8 April 2005; corrected 15 April 2005)

\begin{abstract}
We have studied the discrete electronic spectrum of closed metallic nanotube quantum dots. At low temperatures, the stability diagrams show a very regular fourfold pattern that allows for the determination of the electron addition and excitation energies. The measured nanotube spectra are in excellent agreement with the theoretical predictions based on the nanotube band structure. Our results permit the complete identification of the electron quantum states in nanotube quantum dots.
\end{abstract}

DOI: 10.1103/PhysRevB.71.153402

PACS number(s): 73.22.Dj, 73.63.Fg, 73.23.Hk

Since their discovery ${ }^{1}$ carbon nanotubes (NTs) have emerged as prototypical one-dimensional conductors. ${ }^{2}$ At low temperatures, NT devices form quantum dots (QDs) where single-electron charging and level quantization effects dominate. ${ }^{3,4}$ A continuous improvement in device fabrication and NT quality has enabled the recent observation of twoelectron periodicity in "closed" QDs ${ }^{5}$ and four-electron periodicity in "open" single-wall and multiwall NT QDs., Theoretically, the low-energy spectrum of single-wall nanotube (SWNT) QDs has been modeled by Oreg et al. ${ }^{8}$ Experiments on open NT QDs are compatible with this model, but the presence of the Kondo effect and broadening of the energy levels prevents the observation of the full spectrum. ${ }^{9}$ An analysis of the electronic excitations is therefore still lacking.

The twofold degenerate, low-energy band structure of a metallic SWNT is schematically shown in Fig. 1(a). Quantization along the nanotube axis leads to a set of singleparticle states that are equally spaced because of the linear dispersion relation. ${ }^{10}$ The combination of the two bands and the spin yields a fourfold periodicity in the electron addition energy. The simplest model to describe QDs is the constant interaction (CI) model, ${ }^{11}$ which assumes that the charging energy is constant and independent of the occupied singleparticle states. To describe NT QDs the CI model has been extended ${ }^{8}$ to include five independent parameters: the charging energy $E_{C}$, the quantum energy-level separation $\Delta$, the subband mismatch $\delta$ [see Fig. 1(a)], the exchange energy $J$, and the excess Coulomb energy $d U$. Figure 1(c) illustrates the meaning of the last two parameters. An independent verification of the Oreg et al. model $^{8}$ requires the observation of the ground-state addition energies and of at least two excited states. To the best of our knowledge, such a study has not been reported.

In this paper we investigate the excitation spectrum of closed SWNT QDs. Not only the ground-state, but also the complete excited-state spectrum of these QDs are measured by transport-spectroscopy experiments, enabling us to determine all five parameters independently. With these, the remaining measured excitation energies are well predicted, leading to a complete understanding of the spectrum, without adjustable parameters.

High pressure $\mathrm{CO}$ conversion ${ }^{12}$ (HiPco)- and chemical vapor deposition (CVD)-grown ${ }^{13}$ NTs are used for the fabrication of the devices. HiPco tubes are dispersed from a dichloroethane solution on an oxidized, $p$-doped Si substrate. The
CVD nanotubes are grown from catalyst particles on predefined positions. Individual NTs are located by atomic force microscopy (AFM) with respect to predefined marker positions. Electrodes are designed on top of straight segments of NTs. The highly doped silicon is used as a backgate to change the electrostatic potential of the NT QD [see Fig. 1(b)]. We fabricate NT devices with lengths in between contacts, $L$, varying from $100 \mathrm{~nm}$ to $1 \mu \mathrm{m}$. A four-electron shell filling is observed in over 15 samples. In some cases the fourfold pattern extended over more than 60 electrons added to the QD. Figures 1(d)-1(f) show representative examples of Coulomb blockade (CB) oscillations $^{14}$ in the linearresponse regime. Clearly, the Coulomb peaks are grouped in sets of four, reflecting the twofold character of the NT band structure.

In the following, we focus on three different devices exhibiting similar fourfold periodicity in $\mathrm{CB}$ oscillations. These samples (A, B, and C) have high enough contact resistances so that not only the electron ground states, but also their excited states could be resolved. Together they provide enough information to determine all the parameters in the model. We discuss the results of these three samples separately.

Sample A. This device is made from a HiPco $\mathrm{NT}^{12}$ with $L=180 \mathrm{~nm}$ and a diameter of $1.1 \mathrm{~nm}$ as determined by AFM. It is contacted by evaporating $\mathrm{Cr} / \mathrm{Au}(5 / 75 \mathrm{~nm})$ electrodes. Figure 2(a) shows the current, $I$, as a function of source-drain bias voltage, $V$, and gate voltage, $V_{G}$. In the light-colored diamond-shaped regions, the current is blocked due to $\mathrm{CB}$, and the number of electrons is fixed. The clear fourfold periodicity makes it possible to assign the number of electrons in the last occupied shell. The sizes of the diamonds form an interesting pattern, namely a repetition of small medium small big. This pattern is a consequence of the large subband mismatch compared to the exchange energy, as we show below.

The addition energy is defined as the change in electrochemical potential $\left(\Delta \mu_{N}\right)$ when adding the $(N+1)$ charge to a quantum dot already containing $N$ charges. ${ }^{11}$ The addition energy is obtained by multiplying the diamond width, $\Delta V_{G}$, by a conversion factor, $\alpha(\approx 0.017)$, which relates the gatevoltage scale to the electrochemical potential. ${ }^{14}$

The Oreg model yields the following equations for the addition energy of the $N$ th electron added: ${ }^{15}$ 
a)

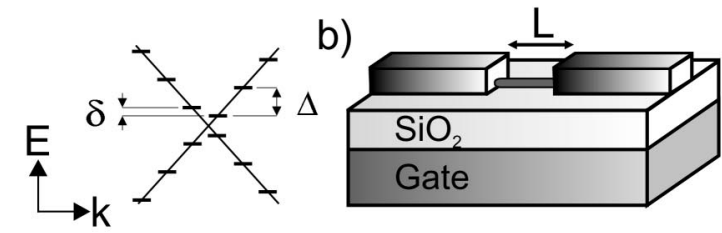

c)

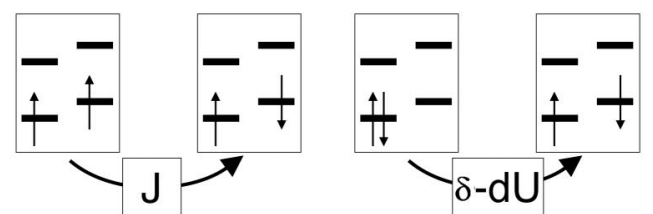

d)

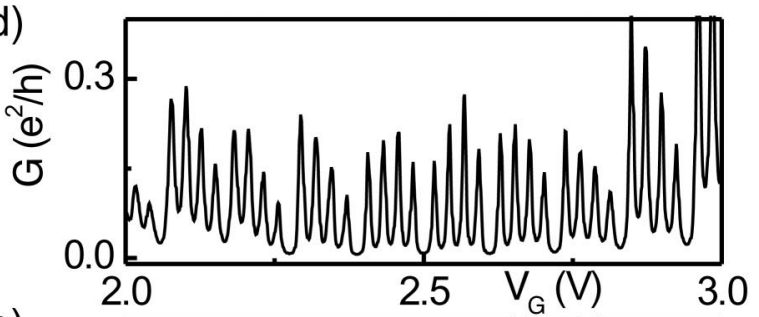

e)

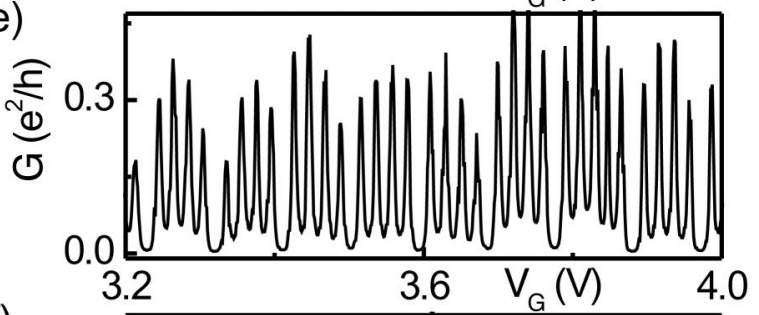

f)

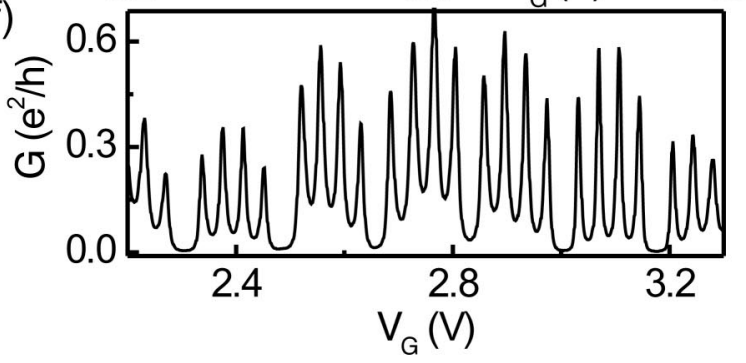

FIG. 1. (a) Low-energy band structure of a metallic SWNT. In a finite-length SWNT, the wave vector $k$ is quantized along the tube axis which leads to a set of quantized energy levels separated by $\Delta$ in each band. $\delta$ is the mismatch between the two bands. (b) Schematic diagram of the device geometry. (c) Meaning of $J$ (left) and $d U$ (right). The exchange energy favors spin alignment and $d U$ is the extra charging energy associated with placing two electrons in the same energy level. (d), (e), and (f) Conductance as a function of gate voltage in the linear-response regime at $4 \mathrm{~K}$ for three different CVD-grown samples. The NT lengths are 500, 680, and $760 \mathrm{~nm}$, respectively.

$$
\begin{gathered}
\Delta \mu_{1}=\Delta \mu_{3}=E_{C}+d U+J, \\
\Delta \mu_{2}=E_{C}+\delta-d U, \\
\Delta \mu_{4}=E_{C}+\Delta-\delta-d U .
\end{gathered}
$$

To extract all five parameters, two more equations are needed. These are provided by the excitation spectrum. In Fig. 2(c) we show the numerical derivative of Fig. 2(a) (i.e., the differential conductance) for the first group of four. The excited states of the electrons are visible for all diamonds.
The value of a particular excitation energy equals the bias voltage at the intersection between the excitation line and the Coulomb diamond edge [see Fig. 2(c)]. The dotted (white) arrows in diamonds one and two in Fig. 2(c) correspond to the first excitation for one and two electrons extra on the NT $\mathrm{QD}$, respectively. The theoretical values of these two energies are

$$
\begin{gathered}
\Delta \mu_{1}^{\mathrm{ex}}=\delta, \\
\Delta \mu_{2}^{\mathrm{ex}}=\delta-J-d U .
\end{gathered}
$$

Equations (1)-(5) allow us to uniquely determine the five unknown parameters from the experimental data alone. We find $E_{C}=4.3 \mathrm{meV}, \Delta=9.0 \mathrm{meV}, \delta=3.2 \mathrm{meV}, J=0.4 \mathrm{meV}$, and $d U \approx 0 \mathrm{meV}$. The values of the parameters do not vary significantly between the different groups, as shown in Fig. 2(b). The theoretically expected value for the level spacing is $\Delta=h v_{F} / 2 L{ }^{3}$ With $v_{F}=8.1 \times 10^{5} \mathrm{~m} / \mathrm{s}$ (Ref. 16) and $L$ $=180 \mathrm{~nm}$, we find $9.3 \mathrm{meV}$, which is in excellent agreement with the experimental value.

Figure 2(d) shows the calculated spectrum of the NT QD using the parameters deduced from the experiment. Some excitations are split by the exchange energy. The stars in the calculated spectrum correspond to the arrows in the experimental data. The excitations denoted with $x$ were used for obtaining the parameters and correspond to the dotted (white) arrows in Fig. 2(c). The calculated spectrum resembles the measured one strikingly well.

Sample B. This sample is CVD grown ${ }^{13}$ with a diameter of $1.3 \mathrm{~nm}$ and $L=500 \mathrm{~nm}$ defined by $\mathrm{Cr} / \mathrm{Au}$ contacts $(5 / 40 \mathrm{~nm})$. After contacting, the entire NT segment between electrodes is suspended by etching away part of the $\mathrm{SiO}_{2} .{ }^{17}$ We have measured the differential conductance, $d I / d V$, as a function of $V$ and $V_{G}$ at $300 \mathrm{mK}$ [Fig. 3(a)]. Again, the regular fourfold patterns are visible in the Coulomb diamonds.

The evolution of the Coulomb peaks as a function of the magnetic field (not shown here) gives information about the spin filling of the states. ${ }^{18}$ We find that the filling is the same as in sample $A$. The excited states of the QD are visible in all groups of four. The model parameters are extracted using the same analysis as described above. The result is shown in Fig. $3(\mathrm{~b})$. The average values are $E_{C}=2.0 \mathrm{meV}, \delta=1.2 \mathrm{meV}, J$ $=0.1 \mathrm{meV}, d U=0.2 \mathrm{meV}$, and $\Delta=3.0 \mathrm{meV}$. The value of $\Delta$ corresponds to a length of $440 \mathrm{~nm},{ }^{3}$ in good agreement with the NT length between contacts. Furthermore, in all groups of four at least one more excitation remains for a comparison between theory and experiment. In all cases we find good agreement. ${ }^{19}$

Sample C. This NT is CVD grown ${ }^{13}$ with a diameter of $2.7 \mathrm{~nm}$ and $L=750 \mathrm{~nm}$. The contacts are made by evaporating Ti/Au (20/40 nm). Figure 3(c) shows $d I / d V$ as a function of $V$ and $V_{G}$. A very regular pattern of Coulomb diamonds with fourfold periodicity is displayed together with the excited states. In addition, up to three inelastic cotunneling lines ${ }^{20}$ are visible [horizontal lines inside the Coulomb diamonds in Fig. 3(c)].

The observation of three equally sized small diamonds and the fact that the excitations have the same energy for all 
a)

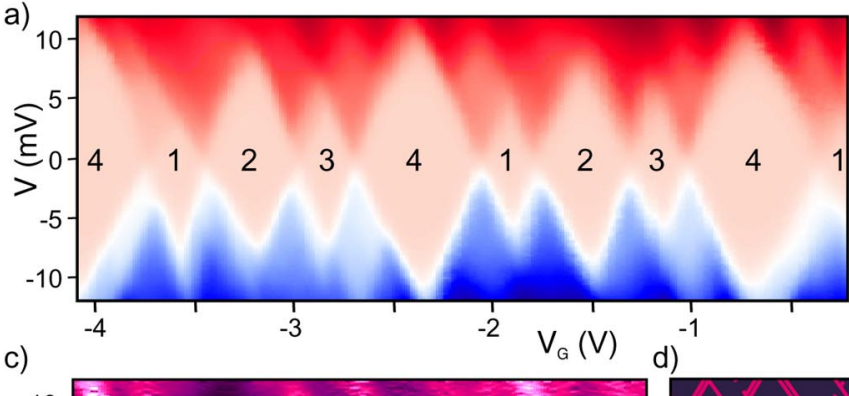

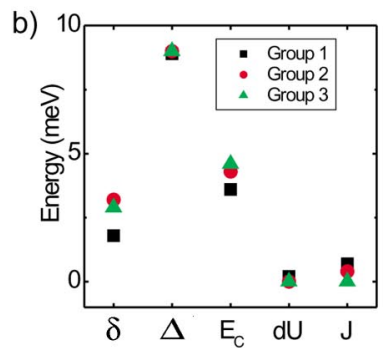
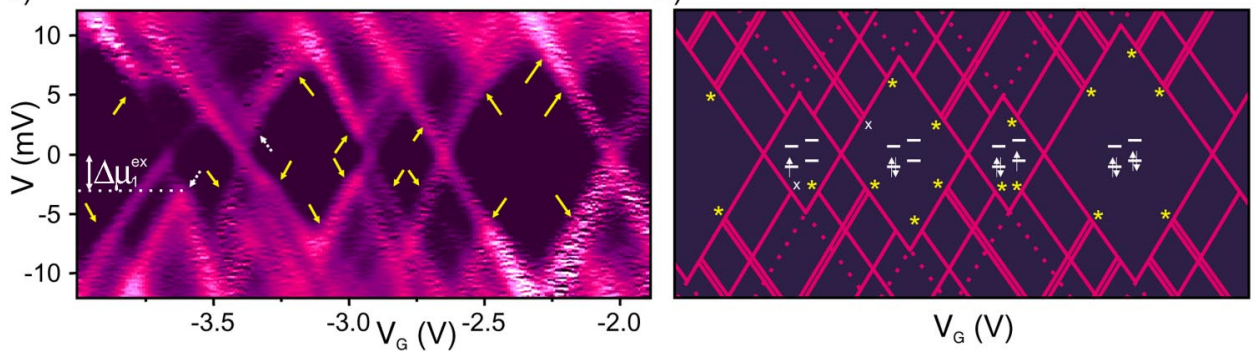

$V_{G}(V)$

FIG. 2. (Color online) Sample A-Current as a function of $V$ and $V_{G}$ at $T=300 \mathrm{mK}$. Current goes from $-40 \mathrm{nA}$ to $+40 \mathrm{nA}$. (b) Values of the parameters for three different groups of four (see text). (c) The differential conductance (dI/dV) for the first group from (a). Black is zero and bright is $>12 \mu \mathrm{S}$. Lines running parallel to the diamond edges correspond to discrete energy excitations. The excitation energies corresponding to the dotted (white) arrows have been used to deduce the model parameters. The predicted excitations are indicated by (yellow) normal arrows. (d) Calculated spectrum for sample A. The stars correspond to the normal (yellow) arrows in (c) and $x$ corresponds to the dotted (white) arrow. The white diagrams indicate the spin filling of the ground state.

four charge states indicate that $\delta \approx J+2 d U$. We find $E_{C}$ $=6.6 \mathrm{meV}, \Delta=8.7 \mathrm{meV}, \delta \approx J=2.9 \mathrm{meV}$, and $d U \approx 0 \mathrm{meV}$. Theoretically, a level separation of $8.7 \mathrm{meV}$ indicates a NT QD length of $\sim 200 \mathrm{~nm}$, while the distance between contacts is $750 \mathrm{~nm}$. This may suggest that sample $C$ consists of a QD with NT leads connecting it to the contacts. This is consistent with the large value for $E_{C}$. Remarkably, all the predicted excitation lines are present in the spectrum. ${ }^{21}$ Therefore all the electron states can be assigned [Fig. 3(d)].
In summary, we have presented a complete analysis of the electronic spectrum in closed NT QDs. Samples with different lengths, production processes (CVD and HiPco), and contact material all exhibited fourfold periodicity in the electron addition energy. The very regular Coulomb traces and stability diagrams enabled the determination of the ground-state and excited-state electron energies. Knowing precisely the spectrum of nanotube quantum dots is of fundamental importance in experiments involving the applica-


d) GS $\vdots 1-E S: 2-E S: 3-E S: 4-E S$

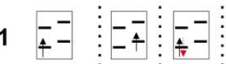

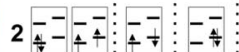

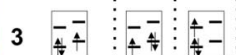

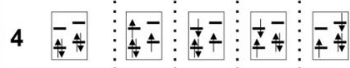

FIG. 3. (Color online) Differential conductance of samples $B$ (a) and $C$ (c) as a function of $V$ and $V_{G}$ measured at $300 \mathrm{mK}$. Black represents $d I / d V \sim 0$, while the lighter tones correspond to a higher conductance. The dashed lines in (c) indicate the excited states together with inelastic cotunneling. (b) Obtained parameters for sample $B$. (d) Electron quantum states of the NT QD. The numbers on the left denote the ground-state (GS) number of electrons in the last occupied shell. The left column indicates the GS electron configuration. (Note that the two-electron GS is degenerate.) The columns on the right denote the excited-state (ES) configuration. Up to four ES's are visible in the large Coulomb diamonds. ${ }^{22}$ The dotted (red) arrow in the second ES for one electron corresponds to an electron excited from the lower shell. 
tion of high-frequency radiation, such as photon-assisted tunneling and coherent control of the electron quantum states.

We thank R. E. Smalley and co-workers for providing the high-quality HiPco nanotubes, and C. Meyer, W. Wetzels, M.
Grifoni, R. Hanson, K.A. Williams, Yu. V. Nazarov, and S. De Franceschi for discussions. Financial support was obtained from the Dutch organization for Fundamental Research on Matter (FOM), which is financially supported by the "Nederlandse Organisatie voor Wetenschappelijk Onderzoek" (NWO).
${ }^{1}$ S. Iijima, Nature (London) 354, 56 (1991).

${ }^{2}$ For reviews, see C. Dekker, Phys. Today 52, No. 5, 22 (1999); P. L. McEuen, Phys. World, June, 31 (2000).

${ }^{3}$ S. J. Tans, M. H. Devoret, H. Dai, A. Thess, R. E. Smalley, L. J. Geerligs, and C. Dekker, Nature (London) 386, 474 (1997).

${ }^{4}$ M. Bockrath, D. H. Cobden, P. L. McEuen, N. G. Chopra, A. Zettl, A. Thess, and R. E. Smalley, Science 275, 1922 (1997).

${ }^{5}$ D. H. Cobden and J. Nygård, Phys. Rev. Lett. 89, 046803 (2002).

${ }^{6}$ W. Liang, M. Bockrath, and H. Park, Phys. Rev. Lett. 88, 126801 (2002).

${ }^{7}$ M. R. Buitelaar, A. Bachtold, T. Nussbaumer, M. Iqbal, and C. Schönenberger, Phys. Rev. Lett. 88, 156801 (2002).

${ }^{8}$ Y. Oreg, K. Byczuk, and B. I. Halperin, Phys. Rev. Lett. 85, 365 (2000).

${ }^{9}$ The Kondo effect shifts the position of the Coulomb peaks towards each other. The level broadening smears out the excitation spectrum.

${ }^{10}$ M. S. Dresselhaus, G. Dresselhaus, and P. C. Eklund, Science of Fullerenes and Carbon Nanotubes (Academic Press, San Diego, 1996).

${ }^{11}$ L. P. Kouwenhoven, D. G. Austing, and S. Tarucha, Rep. Prog. Phys. 64, 701 (2001).

${ }^{12}$ M. J. Bronikowski, P. A. Willis, D. T. Colbert, K. A. Smith, and R. E. Smalley, J. Vac. Sci. Technol. A 19, 1800 (2001).

${ }^{13}$ J. Kong, H. T. Soh, A. M. Cassell, C. F. Quate, and H. Dai,
Nature (London) 395, 878 (1998).

${ }^{14}$ H. Grabert and M. H. Devoret, Single Charge Tunneling (Plenum, New York, 1992).

${ }^{15}$ This set of equations corresponds to the singlet ground state for $N=2$. The triplet case is incompatible with experimental data.

${ }^{16}$ S. G. Lemay, J. W. Janssen, M. van den Hout, M. Mooij, M. J. Bronikowski, P. A. Willis, R. E. Smalley, L. P. Kouwenhoven, and C. Dekker, Nature (London) 412, 617 (2001).

${ }^{17}$ J. Nygård and D. H. Cobden, Appl. Phys. Lett. 79, 4216 (2001).

${ }^{18}$ R. M. Potok, J. A. Folk, C. M. Marcus, V. Umansky, M. Hanson, and A. C. Gossard, Phys. Rev. Lett. 91, 016802 (2003).

${ }^{19}$ The data show a very weak excitation around $200 \mu \mathrm{eV}$. The origin of this excitation might be vibrational [H. Park, J. Park, A. K. L. Lim, E. H. Anderson, A. P. Alivisatos, and P. L. McEuen, Nature (London) 407, 57 (2000)], corresponding to the first longitudinal mode in this suspended NT.

${ }^{20}$ S. De Franceschi, S. Sasaki, J. M. Elzerman, W. G. van der Wiel, S. Tarucha, and L. P. Kouwenhoven, Phys. Rev. Lett. 86, 878 (2001).

${ }^{21}$ The experimental value of the addition energy for the large Coulomb diamond is $\sim 15 \%$ larger than that deduced from the spectrum. The small Coulomb diamonds and the complete excitation spectrum are in perfect agreement with theory.

${ }^{22}$ There is more than one degenerate configuration for some of the excited states. For simplicity we only show one of them. 